\title{
Awake surgery: skills of neurosurgeon matter but those of patient too. How to optimize functional brain mapping by improving per-operatory testing?
}

\author{
Niang $^{1,2}$ \\ Charras $^{1,2}$ \\ Argon $^{3}$ \\ Azevedo $^{1}$ \\ Bonnetblanc ${ }^{1,2}$ \\ Duffau $u^{4,5}$ \\ Guiraud $^{1}$ \\ (1) LIRMM (Montpellier), France \\ (2)INSERM U887, Université de Bourgogne (Dijon), France \\ (3)LEAD (Dijon),France \\ (4) Hôpital Gui de Chauliac (Montpellier), France \\ (5) INSERM U1051, Institut des Neurosciences de Montpellier, (France) \\ E-mail: cheikhniang_4@hotmail.fr,pom.charras@lirmm.fr,stephane.argon@u-bourgogne.fr, \\ christine.azevedo@inria.fr,guiraud@lirmm.fr, h-duffau@,chu-montpellier.fr, \\ francois.bonnetblanc@u-bourgogne.fr
}

\begin{abstract}
It is now possible to perform resections of slowgrowing tumors in awake patients. Using direct electrical stimulation (DES), real-time functional mapping of the brain can be used to prevent the resection of essential areas near the tumor. For now, simple clinical tests are performed on conscious patients and combined with DES in order to discriminate functional and non-functional areas invaded by the tumors. In this work we try to develop a simple device based on a simple technology to better quantify the performances of the patients during the surgery itself and give a real-time feedback to the neurosurgeon that will help to further guide the surgery by improving the sensibility of the functional mapping. This procedure should also allow building a strong database that should serve retrospectively to improve the surgical procedure and reinforce the neurosurgeons' experience as well as to monitor the patients' performances all along their life.
\end{abstract}

\section{Awake surgery of low-grade gliomas: clinical aspects.}

"Low-grade gliomas" (LGG, WHO grade II gliomas) are slow-growing tumors of the CNS. They progress slowly (4 $\mathrm{mm}$ per year on average [1]) for 7-8 years, before undergoing anaplastic transformation. During this slow progression, most LGG patients exhibit normal clinical exam results [2], although mild cognitive disorders can be detected by extensive assessments [3]. LGG resection is subject to two constraints: (i) removing as much of the invaded tissue as possible and (ii) minimizing functional sequelae. During surgical resections, the use of direct electrical stimulation (DES) is widely advocated for functional mapping. This technique causes a transient virtual lesion, allowing the structures that are essential to the function to be identified and preserved at each stage of the resection. The rules guiding this approach are simple: any reproducible functional disturbance induced by DES indicates interruption of the resection ([4], Figure 1). This technique has proved to be effective and more than $90 \%$ of the patients return to normal socio-professional life, with no functional deficit, usually three months after the surgery [5] (Figure 2).

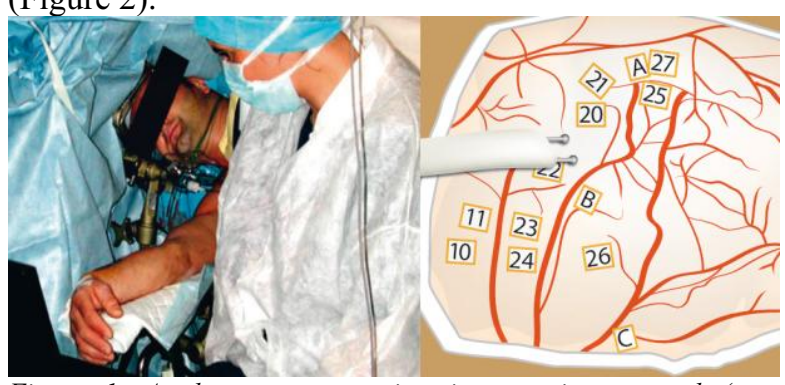

Figure 1. Awake surgery consists in removing tumoral (nonfunctional) tissue when the patient is conscious and is performing some clinical tests (left panel). On the other side of the operatory field (right panel) the neurosurgeon performs functional mapping of the brain using DES and clinical observations given by the speech therapist or the psychologist.

This is an Open Access article distributed under the terms of the Creative Commons Attribution-Noncommercial License 3.0, which permits unrestricted use, distribution, and reproduction in any noncommercial medium, provided the original work is properly cited. 


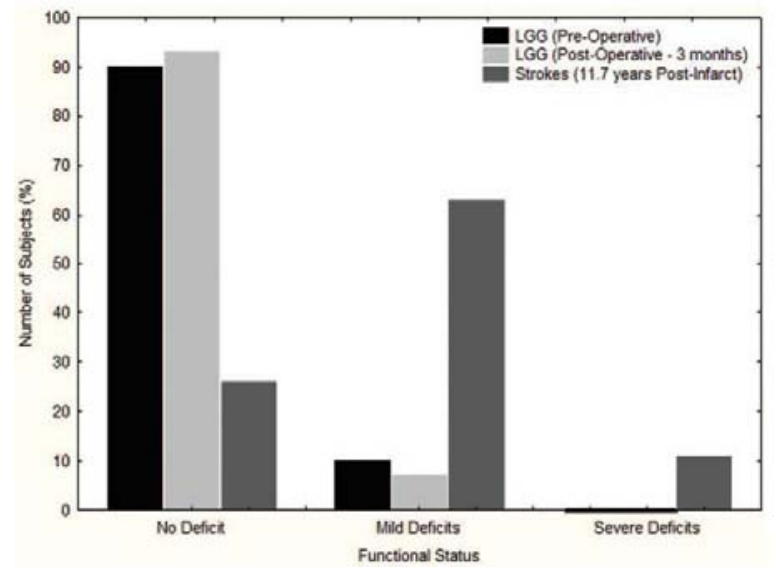

Figure 2. Functional deficits measured on global neurological and rating scales as a percentage of the patient population following $a$ stroke (dark grey) or a slow-growing tumoural invasion (black: LGG patients prior to resection; light-grey: LGG patients 3 months after resection).

In the context of slow-growing injuries, brain reorganization is considerably increased than after acute lesions. The recruitment of remote brain areas in the ipsi- and contralesional hemispheres is much more efficient. Thus, the contrasting recoveries and plastic processes associated with the two types of lesion offer interesting insights that may help understanding brain plasticity [4,6-8]. For slow-growing tumors, the impressive reorganization capacity of the brain allows the subjects to recover much substantially $[4,6,7,9]$.

Some very basic clinical observations indicate that in some cases patients could recover substantially within a single day [10-14]. However, the kinetics of this recovery have not been sufficiently described. Clinical observations reported are at single time points and most are based on global neurological and rating scales.

By contrast, when psychophysical tests are performed on these patients they reveal slight impairments. For instance, awake surgery allows efficient functional recovery but suggests major brain reorganizations which may be accompanied by some slight impairments in particular functional asymmetries between both hemispheres. More particularly, when simple uni and bimanual reaction times (RTs) are compared in a postoperative session after resection in parietal regions (Figure 3), simple RTs revealed functional asymmetries. In particular, longer RTs were observed for the contralesional compared to the ipsilesional hand in the unimanual condition (Figure 4). This asymmetry was reversed for the bimanual condition despite longer RTs. These results are in accordance with changes in brain dynamics, especially between the two hemispheres. In conclusion, it suggests that reaction time paradigms are useful in these patients to monitor functional deficits and to understand brain (re)organization following slowgrowing lesions and awake surgeries.

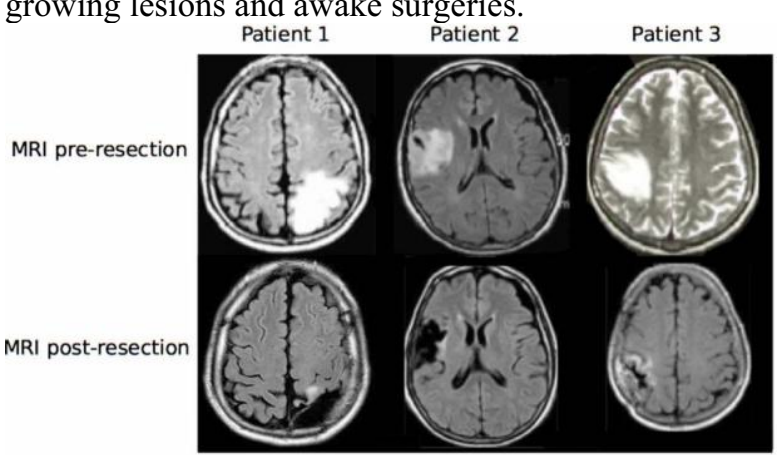

Figure 3. Pre-operative FLAIR-weighted axial and post-operative T1-weighted sagittal MRI for patient PI (left panel), pre- and postoperative FLAIR-weighted axial MRI for P2 (middle panel) and pre-operative T2-weighted axial and post-operative FLAIR-weighted axial MRI for P3 (right panel), showing an invasion (upper row) and a resection (lower row) of the parietal lobe. Neurological conventions (only for this figure).

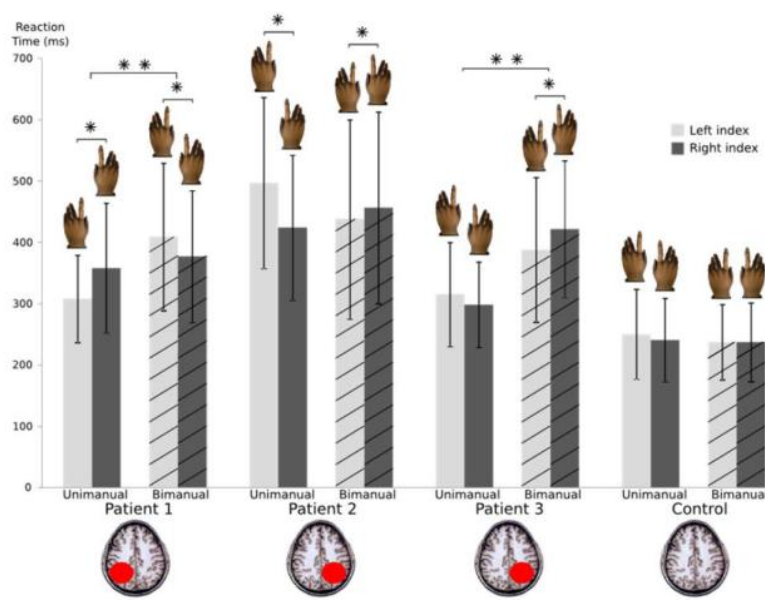

Figure 4. Mean RTs and standard deviation (vertical lines) for the right and left hands in the two unimanual and the bimanual conditions, for P1, P2, P3 and the control group.

In order to better identify functional areas and track functional plasticity in these patients, one solution could be to improve the sensibility of the functional tests that are performed before, during and after the surgery. In consequence, we try to develop a simple device based on a simple technology to better quantify the performances of the patients during the surgery itself and give a real-time feedback to the neurosurgeon that will help to further guide the surgery by improving the sensibility of the functional mapping.

\section{Improving per-operatory testing: simple technical solutions.}

2.1 Mechanical aspects.

In order to perform functional tests during awake surgeries, we built an ambulatory platform allowing to be displaced within the surgical room and nearly from the patient. On this platform, a tactile screen was 
mounted to present various stimuli to the patient and record the patient's response. On the other side of the operatory field, in order to give real time feedback about patients' scores to the neurosurgeon and to pilot the functional tests, a second tactile screen was also positioned and was connected to the patient's interface. Both screens were connected to a central unit and a toolbox also allowed to connect some others electronic devices, to record behavioral responses or electrophysiological signals (all analogical). Importantly, the device is connected to the DES of the neurosurgeon in order to synchronize the stimuli and the patient's response with it.

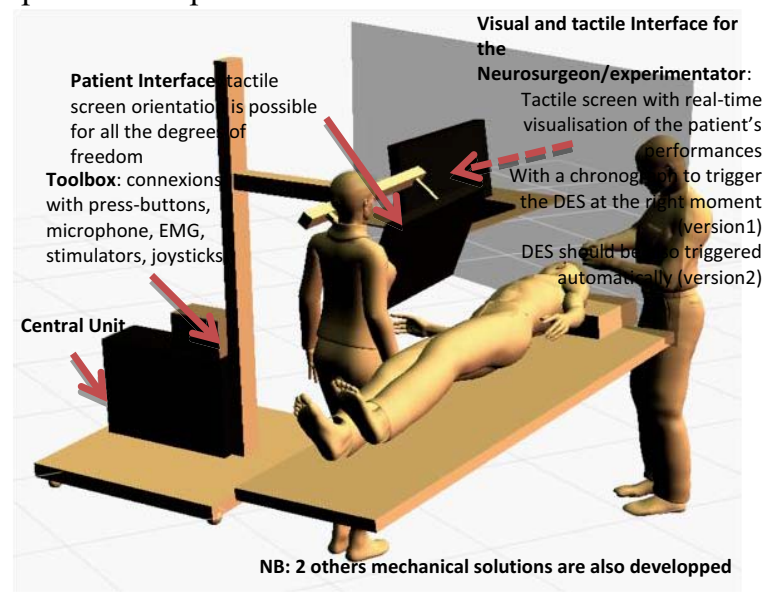

Figure 5. Simplified architecture of the prototype in the context of the surgery room. Interfaces for the Neurosurgeon/Experimentator and for the Patient are shown, as well as the electronic toolbox, all connected to the central unit. Note that DES is not illustrated here, but is also integrated in the whole system and connected to synchronize all signals with it.
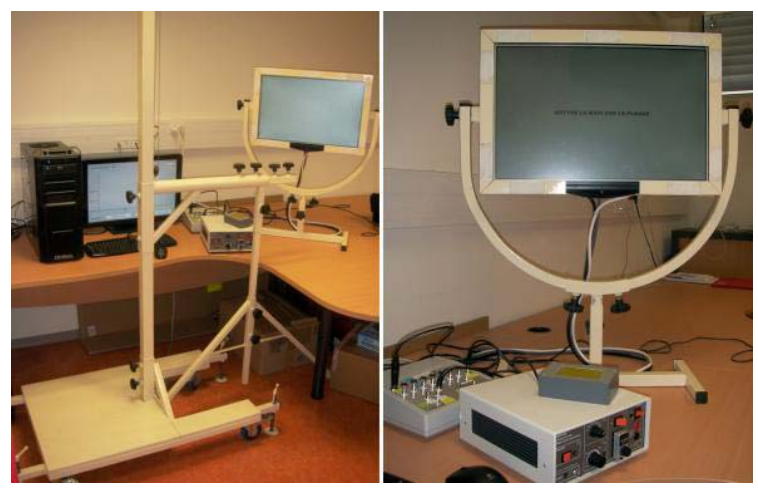

Figure 6. Pictures of the various parts of the whole device (the stimulator used by the neurosurgeon is not shown but replaced here by another similar device).

\subsection{Hardware.}

All devices are connected to a toolbox including an acquisition card which is a bus-powered isolated USB multifunction data acquisition. It allows managing the input and output of all devices used, and provided an isolation CAT I of $60 \mathrm{~V}$ for a precision and an increased safety. The toolbox is connected directly to the PC via an isolator of USB type. All the provisions are taken into account to ensure the safety of the patient during electric stimulations: all devices are electrically isolated from each other. The stimulator itself is electrically isolated.

During the stimulation of the central and peripheral nervous system, the stimulator continuously displays the current delivered and the measured impedance of the current circuit. A TTL signal allowed synchronizing the stimulator with the testing platform. Others devices have been developed by our own such as for instance, a haptic glove in order to evaluate the interaction between the patient's fingers and an object. The aim here is to detect when the patients tighten or slacken the object and to identify precisely hand functional areas when the tumor to be removed is located near the primary motor or sensory areas.

\subsection{Software.}

The software is programmed in $\mathrm{C}++$. The neurosurgeon/experimenter interface is built to pilot the task and to give a real time feed-back to the neurosurgeon about the time of presentation of the different events allowing for the neurosurgeon to trigger the DES in function of these events. The patient interface presents various stimuli in function of the task to be performed. Tasks are selected for their sensibility and in function of the location of the tumors to increase the sensibility of the functional mapping. The temporal precision is estimated to a few milliseconds. Patient's responses are compared with some reference data to determine whether the responses are normal or erroneous. All responses are recorded in a database, easy to export in usual statistical softwares allowing an off-line analysis.

\section{Conclusions.}

This project aims to be able to use psychophysical and neuropsychological paradigms in the surgery room in combination with a simple and modern technology to improve the sensibility and the quality of brain functional mapping. This effort is clearly not independent from the building of selected tasks that are and will be developed. They could be inspired from the scientific literature, with a clear focus on their sensibility. As such they should help the neurosurgeon to make a decision regarding surgical choices. Another, point that should be addressed is about the specification of the DES parameters which are empirically used for now. Indeed, it should be interesting to investigate various forms of electrical currents and determine the most efficient ones in brain functional mapping. Answers to this question are still open. 


\section{References}

[1] Mandonnet, E., Delattre, J. Y., Tanguy, M.L. Swanson, K R, Carpentier, A. F., Duffau, H., et al. Continuous growth of mean tumor diameter in a subset of grade II gliomas. Annals of Neurology 2003, 53: 524-528.

[2] Walker DG, Kaye AH. Low grade glial neoplasms. J Clin Neurosci 2003; 10(1):1-13.

[3] Taphoorn MJ, Klein M. Cognitive deficits in adult patients with brain tumours. Lancet Neurol 2004; 3(3):159-168.

[4] Duffau H. Lessons from brain mapping in surgery for low-grade glioma: insights into associations between tumour and brain plasticity. Lancet Neurol 2005; 4(8):476-486.

[5] Duffau H, Capelle L, Denvil D, et al. Functional recovery after surgical resection of low grade gliomas in eloquent brain: hypothesis of brain compensation. J Neurol Neurosurg Psychiatry 2003; 74(7):901-907.

[6] Bonnetblanc F, Desmurget M, Duffau H. Low grade gliomas and cerebral plasticity: fundamental and clinical implications. Med Sci (Paris) 2006; 22(4):389-394.

[7] Desmurget M, Bonnetblanc F, Duffau H. Contrasting acute and slow-growing lesions: a new door to brain plasticity. Brain 2007; 130(Pt 4):898-914.

[8] Stein DG, Finger S, Hart T. Brain damage and recovery: problems and perspectives. Behav Neural Biol 1983; 37(2): 185-222.

[9] Sallard E, Duffau H, Bonnetblanc F. Ultra fast recovery from a right neglect after an "awake surgery" of a slow growing tumor invading the left parietal area.Neurocase in press. DOI: 10.1080/13554794.2011.556127.

[10] Duffau, H. (2001). Acute functional reorganisation of the human motor cortex during resection of central lesions: A study using intraoperative brain mapping. Journal of Neurology, Neurosurgery and Psychiatry, 70, 506-513.

[11] Duffau, H., \& Capelle, L. (2001a). Functional recuperation following lesions of the primary somatosensory fields. Study of compensatory mechanisms. Neurochirurgie, 47, 557-563.

[12] Duffau, H, \& Capelle, L. (2001b). Functional recuperation after resection of gliomas infiltrating primary somatosensory fields. Study of perioperative electric stimulation. Neurochirurgie, 47, 534-541.

[13] Duffau, H., Sichez, J. P., \& Lehericy, S. (2000). Intraoperative unmasking of brain redundant motor sites during resection of a precentral angioma: Evidence using direct 55 cortical stimulation. Annalsof Neurology, 47, 132-135.

[14] Krainik, A., Lehericy, S., Duffau, H., Capelle, L., Chainay, H., Cornu, P., et al. (2003). Postoperative speech disorder after medial frontal surgery: Role of the supplementary motor area. Neurology, 60, 587-594. 